\title{
Acid-Catalyzed Hydrolysis of tert-Butyl Phenyl Ether. An Application of the Excess Acidity Method
}

\author{
Martti Lajunen $^{\dagger}$ and Kirsi Tanskanen-Lehti \\ Department of Chemistry, University of Turku, FIN-20500 Turku, Finland
}

\begin{abstract}
Lajunen, M. and Tanskanen-Lehti, K., 1994. Acid-Catalyzed Hydrolysis of tertButyl Phenyl Ether. An Application of the Excess Acidity Method. - Acta Chem. Scand. 48: 861-864 (c) Acta Chemica Scandinavica 1994.

Hydrolysis of tert-butyl phenyl ether in aqueous perchloric acid solutions was studied by capillary GC. The activation entropy $\left(67 \mathrm{~J} \mathrm{~K}^{-1} \mathrm{~mol}^{-1}\right)$, the solvent deuterium isotope effect $\left(k_{\mathrm{H}} / k_{\mathrm{D}}=0.37\right)$ and the relative rate $\left(k_{\mathrm{t}-\mathrm{BuOPh}} /\right.$ $\left.k_{\mathrm{iPrOPh}} \approx 10^{7}\right)$ are in agreement with the unimolecular mechanism $(A-1)$ of hydrolysis. The kinetic excess acidity method gives the $\mathrm{p} K_{\mathrm{SH}^{+}}$value as $-1.91 \pm 0.03$ at $283 \mathrm{~K}$ and the $m^{\neq}$parameter as $2.02+0.02$, which also accords only with the $A-1$ mechanism. The values are compared with those evaluated for the hydrolyses of several secondary alkyl phenyl ethers.
\end{abstract}

The hydrolyses of several secondary alkyl phenyl ethers in rather concentrated aqueous perchloric acid solutions were recently studied in our laboratory. ${ }^{1,2}$ The excess acidity method ${ }^{3}$ was separately applied to the measurements of the dissociation constant $\left(K_{\mathrm{SH}^{+}}\right)$of the protonated substrate [eqn. (1)] and to the determination of the kinetic slope parameter $\left(m^{\neq}\right)$, which depends on the mechanism of hydrolysis [eqn. (2)]:

$$
\begin{aligned}
& \log \left(c_{\mathrm{SH}^{+}} / c_{\mathrm{s}}\right)-\log c_{\mathrm{H}^{+}}=m^{*} X_{\mathrm{o}}+\mathrm{p} K_{\mathrm{SH}^{+}} \\
& \log k_{\psi}-\log c_{\mathrm{H}^{+}}-\log \left[c_{\mathrm{S}} /\left(c_{\mathrm{S}}+c_{\mathrm{SH}^{+}}\right)\right]\left(-\log a_{\mathrm{w}}\right) \\
& \quad=m^{\neq} m^{*} X_{\mathrm{o}}+\log \left(k_{\mathrm{o}} / K_{\mathrm{SH}^{+}}\right)
\end{aligned}
$$

In the above equations, $c_{\mathrm{S}}, c_{\mathrm{SH}^{+}}$and $c_{\mathrm{H}^{+}}$indicate the concentrations of the unprotonated and protonated substrate and hydronium ion (i.e. perchloric acid), respectively, and $X_{0}$ stands for the excess acidity ${ }^{4}$ and $a_{\mathrm{w}}$ for the activity of water ${ }^{5}$ in the reaction solution. The term for water activity is included in the case of the bimolecular

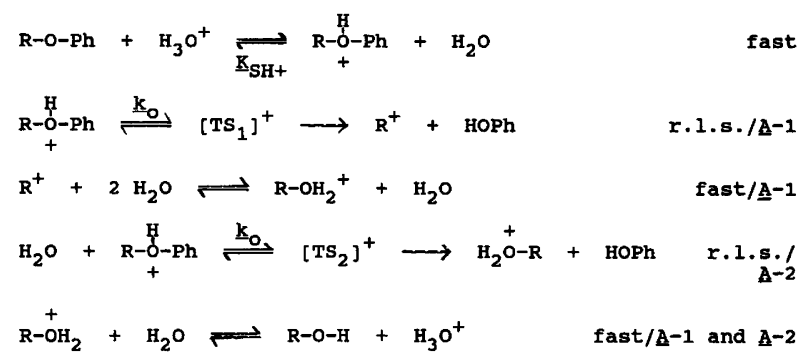

Scheme 1

† To whom correspondence should be addressed. mechanism $(A-2)$ and excluded in the case of the unimolecular mechanism $(A-1)$. The slope parameter $m^{*}$ depends on the site of protonation and $m^{\neq}$on the character of the transition state (Scheme 1; TS = transition state and r.l.s. $=$ rate-limiting stage $)^{3}$

Difficulties appeared, however, in the extrapolations of the $\mathrm{p} K_{\mathrm{SH}^{+}}$values from the lower temperatures, where they were measured, to the higher ones, where the rate constants $\left(k_{\psi}\right)$ for eqn. (2) were measured. ${ }^{1,2}$ The lower temperatures were used for the elimination of a possible hydrolysis of the substrate during the $\mathrm{pK}_{\mathrm{SH}^{+}}$measurements. Therefore it would be easier and time-saving if both $\mathrm{p} K_{\mathrm{SH}^{+}}$and the slope parameters $\left(m^{*}\right.$ and $m^{\neq}$) could be evaluated at the same temperature, and preferably with common measurements. This is possible in theory, if eqns. (1) and (2) are combined to get eqn. (3), in which $k_{\mathrm{a}}=k_{\psi} / c_{\mathrm{H}^{+}}:$

$$
\begin{aligned}
\log k_{\mathrm{a}}\left(-\log a_{\mathrm{w}}\right)= & m^{\neq} m^{*} X_{\mathrm{o}} \\
& -\log \left[1+\left(c_{\mathrm{H}^{+}} / K_{\mathrm{SH}^{+}}\right) 10^{m^{*} \mathbf{X}_{\mathrm{o}}}\right] \\
& +\log \left(k_{\mathrm{o}} / K_{\mathrm{SH}^{+}}\right)
\end{aligned}
$$

Now the parameters $m^{*}, m^{\neq}, K_{\mathrm{SH}^{+}}$and $k_{0}$ (the medium-independent rate constant of the rate-limiting stage) or $\log \left(k_{0} / K_{\mathrm{SH}^{+}}\right)$(the logarithm of the acid-catalyzed rate constant in the extremely dilute acid solution when $X_{0}=0$ ) can be estimated by the aid of a nonlinear leastsquares minimization provided that the experimental data are accurate enough. ${ }^{6}$

The kinetic excess acidity method [eqn. (3)] is applied in the present work to the hydrolysis of tert-butyl phenyl ether. A measurement of its $\mathrm{p} K_{\mathrm{SH}^{+}}$value by the traditional method was not succesful because of its high rate 
of hydrolysis. ${ }^{7}$ The activation parameters, solvent deuterium isotope effect and products of hydrolysis were also determined, and the recent kinetic data for five secondary alkyl phenyl ethers ${ }^{1,2}$ were used to obtain the values of $m^{*}, m^{\neq}$and $\mathrm{p} K_{\mathrm{SH}}$, for the sake of comparison.

\section{Experimental}

Material. tert-Butyl phenyl ether was synthesized by the usual method. ${ }^{8}$ The product ( $36 \%$ of theoretical) was purified by distillation (purity $>99 \%$ by GC) and its ${ }^{1} \mathrm{H}$ and ${ }^{13} \mathrm{C}$ NMR spectra were in agreement with the structure.

Kinetic measurements. The disappearance of the substrate in $\mathrm{HClO}_{4}(\mathrm{aq})$ was followed by GC (an FFAP capillary column) with cyclopentanone as internal standard and methylene chloride as extracting solvent. ${ }^{6}$ The first-order kinetics were always strictly obeyed, and the parallel runs gave rate constants which were equal within the average accuracy of $3 \%$.

Product analysis. The products of hydrolysis were studied by the GC and GC FTIR methods ${ }^{\circ}$ by using tert-butyl alcohol (Merck, 99.5\%), phenol (Merck, 99.5\%) and 2-, 3- and 4-tert-butylphenols (Aldrich, 99\%) as reference compounds.

\section{Results and discussion}

Disappearance rate constants of tert-butyl phenyl ether in aqueous perchloric acid solutions at different temperatures and acid concentrations, as well as in deuterioperchloric acid, are listed in Table 1, together with the activation parameters and solvent deuterium isotope effect. The positive activation entropy $\left(67 \mathrm{~J} \mathrm{~K}^{-1} \mathrm{~mol}^{-1}\right)$ and the isotope effect $\left(k_{\mathrm{H}} / k_{\mathrm{D}}=0.37\right)$ are typical of the unimolecul-
Table 1. Disappearance rate constants of tert-butyl phenyl ether in aqueous perchloric acid at different temperatures and acid concentrations, as well as the activation parameters at $298 \mathrm{~K}$ and the solvent deuterium isotope effect at $293 \mathrm{~K}$.

\begin{tabular}{lllc}
\hline$T / K$ & $\begin{array}{l}c\left(\mathrm{HClO}_{4}\right) / \\
\mathrm{mol} \mathrm{dm}^{-3 a}\end{array}$ & $X_{\mathrm{o}}{ }^{b}$ & $k_{\psi} / 10^{-4} \mathrm{~s}^{-1}$ \\
\hline 283.2 & 0.996 & & $0.71(5)$ \\
293.2 & 0.994 & & $3.84(19)$ \\
298.2 & 0.992 & & $8.00(12)$ \\
298.2 & 1.000 & & $8.3(3)^{c}$ \\
303.2 & 0.990 & & $17.6(8)$ \\
308.2 & 0.987 & & $36.5(7)$ \\
313.2 & 0.986 & & $67.4(17)$ \\
293.2 & 1.245 & & $6.84(14)$ \\
293.2 & 1.246 & & $18.42(16)^{d}$ \\
283.2 & 0.996 & 0.288 & $0.71(5)$ \\
283.2 & 1.526 & 0.411 & $1.86(2)$ \\
283.2 & 2.03 & 0.529 & $4.21(24)$ \\
283.2 & 2.49 & 0.649 & $8.84(4)$ \\
283.2 & 2.80 & 0.733 & $15.08(24)$ \\
283.2 & 3.04 & 0.804 & $22.2(6)$ \\
283.2 & 3.49 & 0.953 & $37.7(5)$ \\
283.2 & 3.99 & 1.136 & $86.2(17)$ \\
283.2 & 4.54 & 1.357 & $219(5)$
\end{tabular}

Kinetic parameters:

$\Delta H^{\neq}=110.7(14) \mathrm{kJ} \mathrm{mol}^{-1}$

$\Delta S^{\neq}=67(5) \mathrm{J} \mathrm{K}^{-1} \mathrm{~mol}^{-1}$

$k_{\mathrm{H}} / \mathrm{k}_{\mathrm{D}}=0.372(11)$

${ }^{a}$ Temperature corrected. ${ }^{b}$ Excess acidity, ${ }^{4}$ temperature corrected. ${ }^{3}{ }^{c}$ Calculated from the activation parameters. ${ }^{d}$ Measured in $\mathrm{DClO}_{4}\left(\mathrm{D}_{2} \mathrm{O}\right)$.

ar hydrolysis mechanism $\left(A-1\right.$; the calculated $k_{\mathrm{H}} / k_{\mathrm{D}}$ limits for this mechanism are 0.3 and 0.5$).{ }^{9}$ The very high relative rate of hydrolysis $\left[k_{\mathrm{t}-\mathrm{BuOPh}} / k_{\mathrm{iPrOPh}} \approx 10^{7}\right.$ in $1 \mathrm{M}$ $\mathrm{HClO}_{4}(\mathrm{aq})$ at $\left.308 \mathrm{~K}\right]^{2}$ also supports this mechanism. ${ }^{10}$ The observed products: isobutene, tert-butyl alcohol, phenol and traces of 2- and 4-tert-butylphenols, are typical

Table 2. Parameters of the excess acidity method evaluated by both the nonlinear least-squares minimization [eqn. (3)] and eqns. (1) and (2) for the hydrolyses of alkyl phenyl ethers ( $\mathrm{R}-\mathrm{O}-\mathrm{Ph})$ in $\mathrm{HClO}_{4}(\mathrm{aq})$. The term for water activity is excluded from the equations.

\begin{tabular}{lllllll}
\hline $\mathrm{R}$ & $\mathrm{T} / \mathrm{K}$ & Eqn. & $m^{*}$ & $\mathrm{p} K_{\mathrm{SH}^{+}}$ & $m^{\neq}$ & $\log \left(k_{\mathrm{o}} / K_{\mathrm{SH}^{+}}\right.$ \\
\hline tert-Bu & 283 & 3 & $1.00(2)$ & $-1.91(3)$ & $2.02(2)$ & $-4.70(1)$ \\
isoPr $^{a}$ & 308 & 3 & $0.98(1)$ & $-6.33(11)$ & $1.44(3)$ & $-9.7(2)$ \\
& $288,308^{b}$ & 1,2 & 1.00 & -6.13 & $1.46(5)$ & $-9.9(2)$ \\
cyclo- $^{\text {Pent }}$ & 279 & 3 & $1.00(2)$ & $-5.94(11)$ & $2.02(3)$ & $-13.0(2)$ \\
cyclo- $^{a}$ & 279 & 1,2 & 0.97 & -5.83 & $2.01(6)$ & $-12.8(3)$ \\
Hex $^{a}$ & 279 & 3 & $1.00(5)$ & $-5.48(30)$ & $2.07(9)$ & $-13.0(2)$ \\
exo-2- $^{\text {NB, }}$ & 279 & 1,2 & 0.96 & -5.76 & $1.93(11)$ & $-13.2(5)$ \\
endo-2- $^{c, d}$ & 308 & 3 & $1.06(2)$ & $-3.74(4)$ & $1.43(2)$ & $-6.42(2)$ \\
NB $^{c, d}$ & 308 & 2 & - & - & {$[1.37(4)]^{e}$} & {$[-6.26(7)]^{e}$} \\
\hline
\end{tabular}

${ }^{a}$ Ref. 2. ${ }^{b}$ Eqn. (1) at $288 \mathrm{~K}$ and eqn. (2) at $308 \mathrm{~K} ; \mathrm{p} K_{\mathrm{SH}^{+}}$assumed to be independent of temperature. ${ }^{c} \mathrm{NB}=$ norbornyl. ${ }^{d}$ Ref. 1. ${ }^{e}$ Calculated by assuming that $m^{*}=1.01$ and $\mathrm{p} K_{\mathrm{SH}^{+}}=-5.56{ }^{1}$ 
of the hydrolysis of alkyl phenyl ethers via carbocations. ${ }^{1,2}$

The excess acidity method ${ }^{3}$ is applied to the hydrolysis of tert-butyl phenyl ether by fitting the experimental $\log k_{\mathrm{a}}$ values $\left(k_{\mathrm{a}}=k_{\psi} / c_{\mathrm{H}^{+}}\right.$; Table 1$)$ according to eqn. (3) versus $X_{0}$ and $c\left(\mathrm{HClO}_{4}\right)$ of the reaction solution by the aid of the nonlinear least-squares minimization using guessed initial values for the parameters and altering them systematically. The averages of the best values with standard deviations are given in Table 2, together with the corresponding parameters re-evaluated by eqn. (3) for the hydrolyses of some secondary alkyl phenyl ethers. ${ }^{1,2}$ The values of $m^{*}$ and $\mathrm{p} K_{\mathrm{SH}^{+}}$as recently measured by employing eqn. (1) and the values of $m^{\neq}$and $\log \left(k_{0} / K_{\mathrm{SH}^{+}}\right)$ as calculated by eqn. (2) are also given for comparison. In the latter cases the correction term, $\log \left[c_{\mathrm{S}} /\right.$ $\left.\left(c_{\mathrm{S}}+c_{\mathrm{SH}^{+}}\right)\right]$, was calculated by using the parameters of eqn. (1).

The value of the parameter $m^{*}$, which depends on the site of protonation, stays practically constant in Table 2 $\left(m^{*}=1.00 \pm 0.03\right)$ and does not markedly differ if it is evaluated by eqn. (1) $\left(m^{*}=0.99 \pm 0.03\right)$ or by eqn. (3) $\left(m^{*}=1.01 \pm 0.03\right)$. Neither does it seem to depend upon the temperature $(279-308 \mathrm{~K})$, which result is similar to those reported earlier. ${ }^{11,12}$

The values of the parameter $m^{\neq}$, which indicates the character of the transition state, are mostly equal in Table 2 within the limits of experimental error if they are calculated by eqn. (2) or (3). They can roughly be divided into two categories: those close to two (av. $2.01 \pm 0.05$ ), which have been measured at 279 or $283 \mathrm{~K}$, and those close to 1.5 (av. $1.48 \pm 0.06$ ), which have been measured at $308 \mathrm{~K}$. They are both in agreement with the $A-1$ mechanism. ${ }^{3}$ It seems possible that $m^{\neq}$decreases with increasing temperature, as was recently observed in the hydration of unsubstituted and substituted norbornenes. ${ }^{12}$ The latter reaction follows the $A-S_{\mathrm{E}} 2$ mechanism (the ratedetermining proton transfer), in which $m^{\neq}$( $=\alpha=$ Brönsted $\alpha)^{3}$ measures the progress of proton transfer from the hydronium ion to the substrate $(0 \leq \alpha \leq 1)$ in the ratelimiting stage of the reaction. Accordingly it is possible that the parameter $m^{\neq}$also depends on the position of the transition state between the protonated substrate and the carbocation intermediate in the $A-1$ mechanism and decreases with increasing temperature, although the change from 2 to 1.5 within ca. $30 \mathrm{~K}$ seems too large. Thus more studies, especially with one substrate at several temperatures, are needed.

The $\mathrm{p} K_{\mathrm{SH}^{+}}$values as calculated from eqn. (1) or (3) in Table 2 do not differ more than $5 \%$ (av. $3 \%$ ), if the measurements were made at the same temperature or at different temperatures (279-308 K). Thus no clear temperature dependence or independence of the basicities of the alkyl phenyl ethers can be seen. However, the $\mathrm{p} K_{\mathrm{SH}^{+}}$values for isopropyl phenyl ether, namely -6.33 at $308 \mathrm{~K}$ and -6.13 at $288 \mathrm{~K}$, presented in Table 2, as well as the value -5.80 at $273 \mathrm{~K}$ measured by Arnett and $\mathrm{Wu},{ }^{7}$ hint at a slight decrease of the basicity with in- creasing temperature, which is opposite to that estimated by eqn. (4): ${ }^{11}$

$\mathrm{p} K_{\mathrm{SH}^{+}, T(2)}=[T(1) / T(2)] \mathrm{p} K_{\mathrm{SH}^{+}, T(1)}$

This equation, in the deprotonation of the protonated substrate, is based on the assumption that $\Delta G^{\circ} \approx \Delta H^{\circ}$, i.e. $\Delta S^{\circ} \approx 0 .^{11}$

The $\mathrm{p} K_{\mathrm{SH}^{+}}$value for tert-butyl phenyl ether $(-1.91$ at $283 \mathrm{~K}$ ) is much less negative than those for several secondary alkyl phenyl ethers (Table 2 and Ref. 7), but in accord with a high basicity predicted by Arnett and $\mathrm{Wu}{ }^{7}$ The reason for this exceptional value is probably the size of the tert-butyl group, which decreases the $\pi$-electron interaction between the oxygen atom and the phenyl group. ${ }^{13}$ Thus its basicity becomes close to those measured for dialkyl ethers. ${ }^{14-16}$

All the kinetic parameters evaluated in Tables 1 and 2 are typical of the $A-1$ mechanism in the hydrolysis of tertbutyl phenyl ether. However, the solvent deuterium isotope effect $[0.37(1)$ at $293 \mathrm{~K}$ ] is practically equal to that $[0.39(2)$ at $298 \mathrm{~K}]$ measured for the $A-2$ hydrolysis of oxetane. ${ }^{6}$ Therefore the effect of water activity (included in the $A-2$ mechanism) is also tested in the case of tertbutyl phenyl ether. By employing the values of $m^{*}$ and $\mathrm{p} K_{\mathrm{SH}^{+}}$in Table 2, the plots of eqn. (2) with and without the term $\log a_{\mathrm{w}}$ are presented in Fig. 1. Both plots are rectilinear ( $r=0.9994$ and 0.9992 , respectively), and thus the linearity does not give any proof of the mechanism, but the slopes, i.e. $m^{\neq} m^{*}, 2.17(3)$ and $2.02(3)$, respectively, and the similar $m^{\neq}$values $\left(m^{*}=1.00\right)$ completely eliminate the $A-2$ mechanism, for which $m^{\neq} \approx 1$ as was recently observed in the hydrolysis of oxetane. ${ }^{6}$

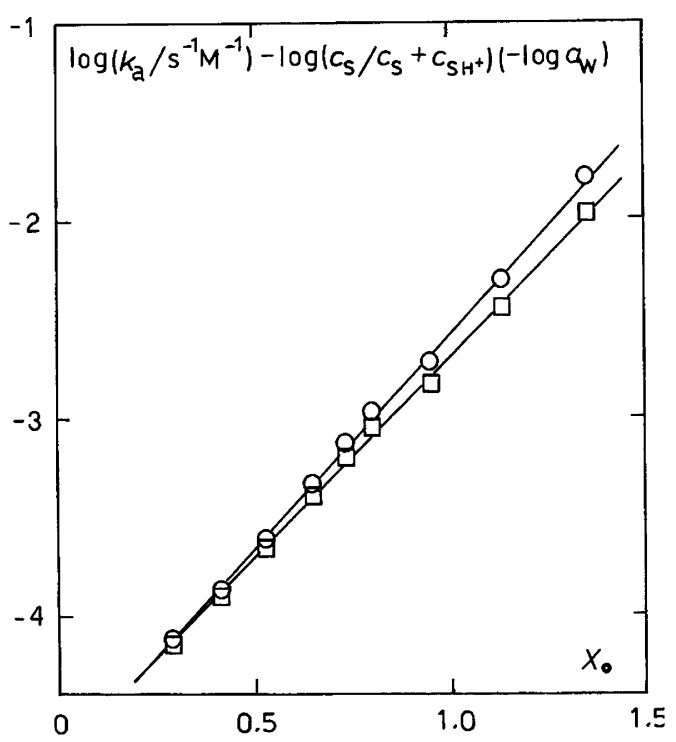

Fig. 1. Excess acidity plots for the hydrolysis of tert-butyl phenyl ether in $\mathrm{HClO}_{4}(\mathrm{aq})$ at $283.2 \mathrm{~K}$ with inclusion $(\mathrm{O})$ and exclusion $(\square)$ of the activity of water (at $298.2 \mathrm{~K}$ ). The straight lines obey eqn. (2). 
The $\mathrm{p} K_{\mathrm{SH}^{+}}$value for exo-2-norbornyl phenyl ether, as evaluated by eqn. (3), is exceptional, because it is two units less negative than that for endo-2-norbornyl phenyl ether (Table 2). If this difference is real, the great rate ratio between the hydrolyses of the exo and endo epimers ( 4170 at $308 \mathrm{~K}$ when $X_{0}=0$ ) is more a result of the higher basicity of the exo epimer $\left(K_{\mathrm{SH}^{+}, \text {endo }} / K_{\mathrm{SH}^{+}, \text {exo }}=98\right)$ than of the faster departure of phenol from the protonated exo substrate $\left(k_{0, \text { exo }} / k_{0, \text { endo }}=43\right)$. This seems strange, ${ }^{17}$ and requires further study.

Acknowledgements. We are grateful to Mr. Antti Hakala, B.Sc., for the program of nonlinear least-squares minimization, to Dr. Esko Taskinen for helpful comments, to Dr. Martti Dahlqvist for recording the GC/FTIR spectra and to Mr. Jaakko Hellman for recording the NMR spectra.

\section{References}

1. Lajunen, M. and Himottu, M. Acta Chem. Scand. 43 (1989) 957.

2. Lajunen, M. and Kähkönen, M. Acta Chem. Scand. 46 (1992) 726
3. Cox, R. A. Acc. Chem. Res. 20 (1987) 27.

4. Cox, R. A. and Yates, K. Can. J. Chem. 59 (1981) 2116.

5. Gordon, A. J. and Ford, R. A. The Chemist's Companion, Wiley-Interscience, New York 1972, pp. 65-66.

6. Lajunen, M. and Koskinen, J.-M. Acta Chem. Scand. 48 (1994) A 2678

7. Arnett, E. M. and Wu, C. Y. J. Am. Chem. Soc. 84 (1962) 1684.

8. Sahyun, M. R. V. and Cram, D. J. Org. Synth. 45 (1965) 89.

9. Lajunen, M. and Uotila, R. Acta Chem. Scand. 46 (1992) 968.

10. Bentley, T. W., Bowen, C. T., Parker, W. and Watt, C. I. F. J. Chem. Soc., Perkin Trans. 2 (1980) 1244.

11. Cox, R. A. and Yates, K. Can. J. Chem. 62 (1984) 2155.

12. Lajunen, M., Virta, M. and Kylliäinen, O. Acta Chem. Scand. 48 (1994) 122.

13. Kalabin, G. A., Kushnarev, D. F., Valeyev, R. B., Trofimov, B. A. and Fedotov, M. A. Org. Magn. Reson. 18 (1982) 1.

14. Arnett, E. M. and Wu, C. Y. J. Am. Chem. Soc. (a) 82 (1960) 4999; (b) 84 (1962) 1680.

15. Perdoncin, G. and Scorrano, G. J. Am. Chem. Soc. 99 (1977) 6983.

16. Bellon, L., Taft, R. W. and Abboud, J.-L. M. J. Org. Chem. 45 (1980) 1166.

17. Lenoir, D., Apeloig, Y., Arad, D. and Schleyer, P. v. R. J. Org. Chem. 53 (1988) 661 and references cited therein.

Received March 18, 1994. 